\title{
Ecosystem structure, function, and composition in rangelands are negatively affected by livestock grazing
}

\author{
David J. Eldridge, ${ }^{1,2,4}$ Alistair G. B. Poore, ${ }^{1}$ Marta Ruiz-Colmenero, ${ }^{1}$ Mike Letnic, ${ }^{2}$ and Santiago Soliveres ${ }^{3}$ \\ ${ }^{1}$ Evolution and Ecology Research Centre, School of Biological, Earth and Environmental Sciences, University of New South \\ Wales, Sydney, New South Wales 2052 Australia \\ ${ }^{2}$ Centre for Ecosystem Science, School of Biological, Earth and Environmental Sciences, University of New South Wales, \\ Sydney, New South Wales 2052 Australia \\ ${ }^{3}$ Institute of Plant Sciences, University of Bern, Altenbergrain 21, 3013 Bern, Switzerland
}

\begin{abstract}
Reports of positive or neutral effects of grazing on plant species richness have prompted calls for livestock grazing to be used as a tool for managing land for conservation. Grazing effects, however, are likely to vary among different response variables, types, and intensity of grazing, and across abiotic conditions. We aimed to examine how grazing affects ecosystem structure, function, and composition. We compiled a database of 7615 records reporting an effect of grazing by sheep and cattle on 278 biotic and abiotic response variables for published studies across Australia. Using these data, we derived three ecosystem measures based on structure, function, and composition, which were compared against six contrasts of grazing pressure, ranging from low to heavy, two different herbivores (sheep, cattle), and across three different climatic zones. Grazing reduced structure (by $35 \%$ ), function (24\%), and composition (10\%). Structure and function (but not composition) declined more when grazed by sheep and cattle together than sheep alone. Grazing reduced plant biomass $(40 \%)$, animal richness $(15 \%)$, and plant and animal abundance, and plant and litter cover $(25 \%)$, but had no effect on plant richness nor soil function. The negative effects of grazing on plant biomass, plant cover, and soil function were more pronounced in drier environments. Grazing effects on plant and animal richness and composition were constant, or even declined, with increasing aridity. Our study represents a comprehensive continental assessment of the implications of grazing for managing Australian rangelands. Grazing effects were largely negative, even at very low levels of grazing. Overall, our results suggest that livestock grazing in Australia is unlikely to produce positive outcomes for ecosystem structure, function, and composition or even as a blanket conservation tool unless reduction in specific response variables is an explicit management objective.
\end{abstract}

Key words: ecosystem function; grazing; livestock; plant composition; plant production; structuralmetrics.

\section{INTRODUCTION}

Livestock grazing is one of the most extensive land uses on Earth and an important biotic process affecting plant and animal communities and ecosystem functions. The economies of many people, particularly from rural areas, depend on the provision of goods and services such as milk, meat, wool, and hide derived from livestock. The economic importance of grazing and the reports of positive or neutral effects of grazing on species richness in specific studies (e.g., Socher et al. 2013, Fensham et al. 2014, Kimuyu et al. 2014) has led some to suggest that introducing or reintroducing commercial herds of livestock to intermittently grazed or ungrazed areas (e.g., alpine high country of Australia; Williams et al. 2006) might have benefits for biodiversity and ecosystem functioning (Lunt et al. 2007, Williamson et al. 2014).

Manuscript received 3 July 2015; revised 9 October 2015; accepted 5 November 2015. Corresponding Editor: D. Brunton.

${ }^{4}$ E-mail: d.eldridge@unsw.edu.au
The notion of using livestock grazing to enhance ecosystem functions contrasts markedly with the extensive body of literature on the negative effects of grazing on soil, plant, and animal attributes worldwide. Grazinginduced habitat modification alters species composition by reducing the diversity of plants and terrestrial invertebrates, small mammals, birds, reptiles, and soil crusts (e.g., Williams et al. 2008, van Klink et al. 2014). Grazing also alters community structure by influencing, for example, the return interval of wildfires and the accumulation of flammable fuel (Kimuyu et al. 2014) or plant community composition (Lunt et al. 2012, Fensham et al. 2014). These direct structural and compositional shifts have often large, indirect effects on ecosystem functions. The most obvious functional effect is a direct reduction in net primary productivity through herbivory (Milchunas et al. 1988), resulting in reduced decomposition and changes in the amount and distribution of litter and dung. Grazing also compacts soils, increases erosion, and alters soil hydrological processes (Lunt et al. 2007). Together these direct and indirect effects of 
livestock grazing can have pronounced legacy effects on soils and landscapes that diminish their capacity to maintain key ecological processes, such as decomposition and nutrient flows (Lunt et al. 2007).

The effects of grazing are largely driven by four main factors: (1) the type of herbivore (e.g., Kimuyu et al. 2014), (2) the intensity of grazing pressure (Lunt et al. 2007, Eldridge et al. 2011), (3) the level of plant productivity (e.g., Senft et al. 1987, Proulx and Mazumder 1998), and (4) the evolutionary history of grazing (Milchunas and Lauenroth 1993). First, grazing effects are known to vary between native and domestic herbivores (Riginos and Grace 2008) and among different breeds of livestock, which have different foraging behaviors and patch preferences (Squires 1981). Grazing by sheep, for example, has been associated with increases in plant richness, but cattle grazing can substantially reduce plant diversity or ecosystem functioning (Letnic 2004, Socher et al. 2013). Second, low levels of grazing are likely to induce biotic shifts in communities, mainly by altering composition through increases in diversity (e.g., Lunt et al. 2007, Dorrough et al. 2012, Borer et al. 2014). Moderate to heavy levels of grazing or prolonged use, however, are likely to induce abiotic changes, which are typically associated with reduced soil structure and function (Eldridge et al. 2013).

Despite substantial research using manipulative and mensurative experiments, however, the level of grazing that optimizes livestock productivity and ecosystem richness and functions remains largely elusive. This might be related to the fact that grazing effects also depend on site-level productivity. While there is an increasing body of evidence supporting the notion that low productivity (arid) sites will be more sensitive to grazing (e.g., Proulx and Mazumder 1998, Cingolani et al. 2005), herbivory may be also influential in more productive systems (Milchunas and Lauenroth 1993). The effects of livestock grazing on ecosystem properties and processes are also highly dependent on the evolutionary history of grazing by large herbivores (Milchunas and Lauenroth 1993, Cingolani et al. 2005). Australia has a very short history of grazing by European domestic livestock $(<200 \mathrm{yr})$ and therefore livestock have not co-evolved with the existing vegetation. Prior to the introduction of livestock, Australia supported extensive but low densities of mammalian herbivores, such as kangaroos, whose densities were low because of sparse and unreliable water supplies and predation by dingoes (Letnic et al. 2009).

We use a meta-analytical approach to understand how grazing influences key ecosystem processes and products using 6920 separate observations of the effects of grazing by livestock (sheep, cattle) from 217 studies. Our approach is novel because it uses an average value of response ratios, allowing us to pool attributes that would otherwise occur at different spatial or measurement scales, into three meaningful attributes related to landscape architecture (structure), ecosystem signatures (composition), and how the system supports and maintains critical ecosystem processes (functions). This approach allows the pooling of data across a range of seemingly disparate attributes to arrive at a meaningful scaled up assessment of the response of ecosystems to grazing by livestock and residual (free-ranging and largely uncontrolled) herbivores. The approach has been used to examine the ecosystem effects of shrub encroachment on structural, functional, and compositional attributes in global studies (Eldridge et al. 2013). Our study examines grazing effects across a wide range of environmental conditions (from arid to humid and sub-humid environments) over a large area of Australia. Previous studies have described the effects of grazing on richness (Proulx and Mazumder 1998, van Klink et al. 2014), or changes in structure and composition in response to cattle grazing in arid environments (Letnic 2004). However, we are unaware of any comprehensive meta-analyses that have attempted to assess grazing effects across such a wide range of possible response variables, grazing types, and environmental conditions. A comprehensive understanding of when and how grazing has positive effects on ecosystems and how grazing affects different response variables is required in order to manage these systems more effectively, particularly where it is advocated as a tool to manage for conservation (Lunt et al. 2007).

The Australian continent is an excellent study system in which to assess the effect of grazing on ecosystem structure, function, and composition for a number of reasons. Firstly, grazing by domestic livestock occurs over more than half of its land mass (Fensham et al. 2014). Secondly, it provides a unique opportunity to assess the role of grazing in a system less likely to be confounded by evolutionary changes in plant communities in response to livestock grazing (Dorrough et al. 2012). In contrast to other continents, Australia has a very short history of grazing by European domestic livestock ( $<200 \mathrm{yr}$; Lunt et al. 2012), but a rich history of grazing by a range of macropods (e.g., Macropus spp.) and megafaunal browsing during the Quaternary period. Thirdly, provincial governments across the country are under increasing pressure to allow grazing within conservation reserves from which livestock have long been excluded (Lunt et al. 2007, Williamson et al. 2014). Thus, it is crucial to determine the effect that different levels of grazing (even from ungrazed to low or moderately grazed) have on its ecosystem structure and functioning.

We had three predictions. Firstly, we expected that the amount of change (increase or decrease of the grazing response ratio) in the three community attributes would increase with increases in grazing pressure, i.e., as relative differences in grazing increased. Secondly, we expected that grazing effects on structure, function, and composition would differ among the livestock groups (sheep, cattle, mixed sheep-cattle), given the differences in foraging behavior of the three groups (Squires 1981). 
Thirdly, we predicted that grazing effects would be more pronounced in areas of low productivity (arid to semiarid) than areas of greater productivity (humid to sub-humid) as the former are more sensitive to disturbances (Cingolani et al. 2005, Lezama et al. 2014).

\section{Methods}

\section{Database construction}

We performed a systematic search of the scientific literature to identify quantitative evidence of the effects of grazing by vertebrates on multiple measures of ecosystem composition, structure, and/or functioning. We used the ISI Web of Knowledge (Thomson Reuters, New York, New York, USA) database (1945-2013 period) using the keywords grazing, and Australia. We used published and unpublished reports, articles, reviews, data from student theses, and unpublished data from colleagues who have been monitoring changes inside and outside grazing exclosures across a wide range of rainfall regimes. Studies were only included in our quantitative synthesis if they reported quantitative results of experiments or trials conducted under natural field conditions. Studies involving improved pasture were not included, nor were studies that only reported effects under a nonspecific grazing level and therefore from which we could not derive a grazing response ratio (see Methods: Measurements of grazing intensity).

We recorded the location of the study (state, local site name, latitude, and longitude) and climatic variables (mean annual rainfall and temperature). If not presented in the original publication, data on temperature and rainfall were derived from relevant long-term databases from the Australian Bureau of Meteorology (available online).$^{5}$ From these data, we derived an aridity index $(\mathrm{AI}=$ precipitation/potential evapotranspiration $)$, which ranges from 0.05 to 0.65 (UNEP 1992). This aridity index was transformed to an aridity measure (1-aridity index) to improve the interpretation (higher values mean greater water shortage). In those studies, reporting data for multiple points in time, the results were averaged across years. If several studies presented results in the same experimental plots, only the results of the most recent study were used (Piñeiro et al. 2013). Results presented as graphics were extracted using Datathief (Tummers 2006).

Overall, we compiled a database of 6920 records of an effect of grazing on 278 biotic and abiotic response variables from 217 studies. From this large database, we constructed a set of 4668 independent grazing contrasts; each contrast derived by comparing two levels of grazing for a given response variable. Many studies reported several levels of grazing (e.g., ungrazed, lightly or moderately grazed, heavily grazed) and with multiple response variables (e.g., plant biomass, plant richness, soil carbon), or the experiment was conducted at more than one

\footnotetext{
${ }^{5}$ www.bom.gov.au
}

independent location. In these cases, each contrast between grazing levels for a given response variable or case study provided a separate measure of grazing effect size, but were labelled by study to account for the nonindependence of measures within a study (see Quantifying grazer impacts). We retained all measures from a study as separate observations in order to ensure that our results were as general as possible (Piñeiro et al. 2013). This approach tends to reduce the overall heterogeneity when estimating effect sizes, excluding multiple results from one data source can underestimate such sizes (Gurevitch and Hedges 1999). This approach has been applied widely in many previous ecological meta-analyses (Piñeiro et al. 2013).

Most grazing records were from arid and semi-arid environments, defined as aridity classes $0.03-0.2$ and $0.2-0.5$, respectively (Appendix S1; median rainfall, $320 \mathrm{~mm}$; mean $\pm \mathrm{SD}, 408 \pm 279 \mathrm{~mm}$; range, 120-2447 mm) and the number of records declined substantially with increases in average annual rainfall $\left(F_{1.22}=69.3, P<0.001, R^{2}=0.75\right)$. Few records $(2.5 \%)$ were from sites receiving $>1000 \mathrm{~mm}$ rainfall (Appendix S1). Sixty-percent of records were from eastern Australia (NSW, ACT, Queensland, Victoria; Fig. 1), and almost two-thirds $(62 \%)$ of all records examined grazing by sheep (Appendix S1).

\section{Measurements of grazing intensity}

We extracted quantitative and/or qualitative information on grazing from each study to derive four qualitative levels of livestock grazing (ungrazed, low, medium, and heavy; see Appendix S2). We used the authors' assessment of grazing intensity and validated this with 3134 grazing records for which we had data on both the intensity category and a quantitative measure of grazing, in dry sheep equivalents (Appendix S2). We also recorded the type of grazing animal (e.g., sheep, cattle, goats).

\section{Response variables}

The first set of analyses was conducted to test the overall effect of livestock grazing on the broad ecosystem attributes: structure, composition, and function, as defined by Noss (1990). Structural attributes included a range of variables that represent the physical architecture and spatial arrangement of ecosystems. These variables, which included plant density, cover, patchiness, and patch size and area relationships, are correlated. They are useful predictors of the capacity of landscapes to capture and retain resources Tongway (1995) and their ability to provide the elements needed to sustain specific organisms (habitat quality, e.g., van Klink et al. 2014). For the attribute composition, the variables included in our analyses represented ecosystem signatures relating to the number of species or variety of species within ecosystems. These included measures of species diversity, taxon richness, diversity, similarity, abundance, and 

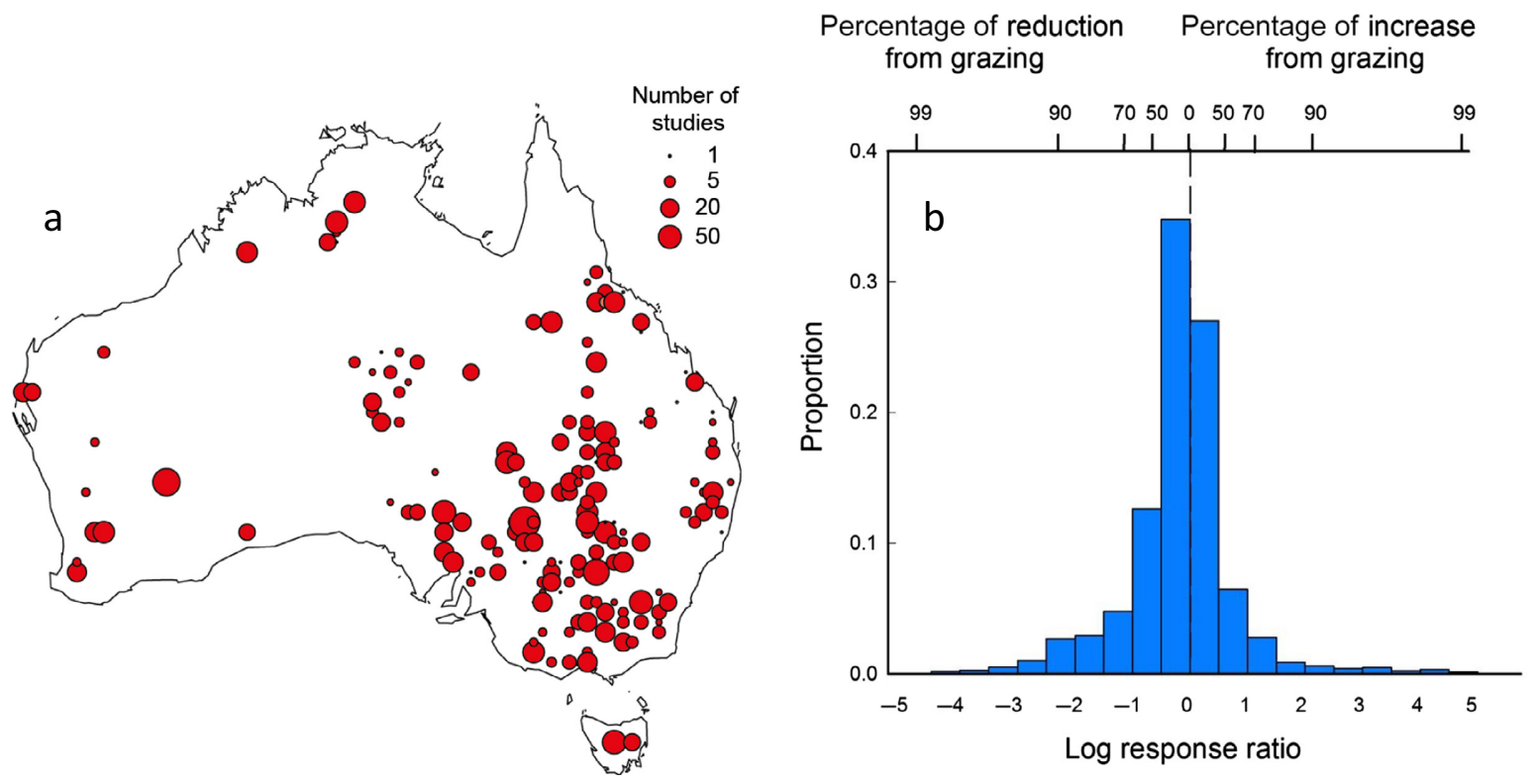

FIG. 1. (a) Map of Australia showing the location and number of studies used in the analyses and (b) histogram showing the frequency distribution of all effect sizes (the log response ratio). Note the higher proportion of log response ratios that show a reduction due to grazing.

frequencies of different biota. These measures again are known to be correlated and are widely used in studies investigating the impacts of livestock grazing (Landsberg et al. 2003). The attribute function is concerned with the fluxes of energy and matter within ecosystems. Variables considered in our analyses for this attribute included biotic-abiotic surrogates of important ecological processes and functions, such as nutrient cycling (e.g., carbon, nitrogen, phosphorus), hydrological processes (e.g., water infiltration, soil moisture), geomorphological processes (e.g., sediment detachment), and production (e.g., net primary productivity, standing biomass, plant decomposition; Noss 1990). Although increases in the log response ratios for most variables indicated an improvement in ecosystem structure, function, or composition, increases in some (e.g., soil erosion, runoff) are equivalent to reduced function. In these cases, the response ratio was transformed by multiplying it by -1 to improve the interpretation (greater score equates with higher function).

The aim of the first set of analyses was to provide generalizable results applicable to a broad range of categories and nuances within each management objective. We did not expect different variables within the three broad categories to respond similarly to grazing, as idiosyncratic responses to grazing have been previously observed for different variables related to ecosystem structure, composition, or function. For example, plant diversity could increase under moderate levels of grazing, whereas mammal diversity could decline under any levels of livestock grazing, and both were included within the composition category. These idiosyncrasies were then considered in subsequent analyses (see Methods: Effects of grazing on plants, animals and soil below), where we investigated the responses to livestock grazing of specific measurements in cases where sample sizes were adequate.

\section{Quantifying grazer impacts}

Effect sizes for the contrasts between the four different levels of grazing were calculated using mean data for a given response variable for each possible comparison between ungrazed, light, medium, and heavy grazing. The effect was estimated as the natural logarithm (ln) of the response ratio $(\mathrm{RR})$

$$
\operatorname{lnRR}=\ln \left(X_{\mathrm{L}} / X_{\mathrm{H}}\right),
$$

where $X_{\mathrm{L}}$ is the mean value of the response variable at the lowest level of grazing and $X_{\mathrm{H}}$ is that value for the highest level. This gave us three values; where low, medium, and heavy grazing were each compared with an ungrazed situation. We also calculated the $\ln R \mathrm{R}$ for three additional comparisons, where these data were available: low compared with medium grazing, low compared with heavy grazing, and medium compared with heavy grazing. The log response ratio is negative when the value of a given response variable is lower as a result of a greater level of grazing.

Although response ratios cannot be estimated when the mean for one level is zero (Dorrough et al. 2012), elimination of such data could limit our ability to detect useful effects of grazing on some response variables with infrequent or low values. If the mean values of any 
treatments were zero (e.g., no plants found in a heavily grazed plot), we substituted zero with the minimum value that was likely to be detected with the sampling method used (e.g., a count of 1 when numbers of individuals per plot was measured, $1 \%$ cover when percent cover was measured; Poore et al. 2012). The possibility of publication biases was examined by inspection of the funnel plot of effect sizes vs. sample size. These did not indicate any publication biases as would be expected in nonsignificant results with low replication were unlikely to be published (Møller and Jennions 2001). Like several recent meta-analyses (e.g., Mooney et al. 2010), we took the conservative approach of not weighting effect sizes by their variance.

\section{Structural, functional, and compositional changes in response to grazing}

We tested our first hypothesis by contrasting the effect sizes for each of composition, structure, and function against our six grazing contrasts, from the least (low vs. ungrazed) to the greatest (heavy vs. ungrazed). We used linear mixed models with $\operatorname{lnRR}$ as the dependent variable. Grazing contrast was a fixed effect and individual study a random effect (accounting for the nonindependence of multiple measures that arose in each study). Linear mixed models were run in the package lme 4 in R (Bates et al. 2014), with the significance of fixed factors tested by likelihood ratio tests. Estimates of $\ln R R$ for each level of the categorical fixed factors were derived from REML and 95\% confidence intervals for these estimates obtained from the likelihood profile. We then tested effects of grazing on the three metrics by examining changes in composition, structure, and function using similar models, but with two separate analyses (1) using low, moderate, and heavy levels vs. ungrazed, and (2) the three different levels of grazing (i.e., low vs. medium, medium vs. heavy, low vs. heavy). We expected that grazing effects on structure, function, and composition would differ among sheep and cattle, and examined this by partitioning grazing effects among three different herbivore groups; sheep, cattle, and mixed sheep and cattle, and used the six different grazing contrasts described previously. To test these, we used a linear mixed model with response type (structure, function, or composition) and grazer contrast as fixed factors and individual study as a random factor.

\section{Effects of grazing on plants, animals, and soil}

We contrasted the magnitude of potential grazing effects among eight attributes for which we had sufficient data (plant litter, cover, biomass, richness and abundance, animal richness and abundance, soil function). We used linear mixed models with attribute type fixed and study a random factor. Soil function included those attributes associated with the cycling of carbon, nitrogen, and phosphorus (e.g., labile carbon, organic carbon, mineralisable nitrogen, available phosphorus), as well as cation exchange capacity. This allowed us to examine potential effects of particular ecosystem attributes that might otherwise be obscured by the previous analyses focusing on broader functional, compositional, and structural metrics.

\section{Effects of aridity on plant, animal, and soil responses to grazing}

We anticipated that grazing effects might be more pronounced and thus more negative in drier (arid, semi-arid) areas (Proulx and Mazumder 1998). We tested this using the index of aridity with separate linear mixed models using grazing contrast and aridity (as a continuous variable) as fixed factors and study as a random effect, to examine whether effects on the eight attributes varied across different aridity zones. Aridity was used because it has predefined classes that are readily interpretable, i.e., $<0.04$ is hyper arid, $0.03-0.2$ is arid, $0.2-0.5$ is semiarid, and $>0.5$ is dry sub-humid to humid (UNEP 1992).

\section{RESUlts}

\section{Structural, functional, and compositional changes in response to grazing}

Averaged across all grazing contrasts, the three ecosystem measures (structure, function, and composition) were negatively affected by grazing (values of the log response ratios less than zero; Fig. 2). When we examined the effects of increasing grazing pressure on structure, function, and composition two trends emerged: (1) a consistent reduction in structure and function for all grazing contrasts, even at the most benign grazing contrast (i.e., ungrazed vs. low grazing), and (2) a marked decline in function at the highest grazing contrast (i.e., ungrazed cf. heavy grazing) compared with the remaining grazing comparisons (Fig. 2). The differences between the effect of grazing for the six grazing contrasts varied among the three ecosystem measures (ecosystem measure by grazing contrast interaction: $\chi^{2}=40.1, \mathrm{df}=10, P<0.001$ ). This interaction was best explained as a generally negative and consistent decline in function, a generally negative to neutral effect on composition, but an increasingly negative decline in structure with increasing grazing contrast (Fig. 2). These trends were similar when we separated the six grazing contrasts into two separate analyses based on (1) the three grazed-ungrazed comparisons (i.e., ungrazed cf. light, medium, or heavy), and (2) the three intermediate levels of grazing (i.e., light cf. moderate, light cf. heavy, moderate cf. heavy; Appendix S3).

Averaged across all three ecosystem measures, the effect of grazing varied significantly among herbivore groups $\left(\chi^{2}=29.0\right.$, df $\left.=2, P<0.001\right)$. For measures of composition, the effect of grazing did not vary among the three herbivore groups (sheep, cattle, sheep + cattle; $\left.\chi^{2}=2.05, \mathrm{df}=2, P=0.57\right)$. However, measures of both 


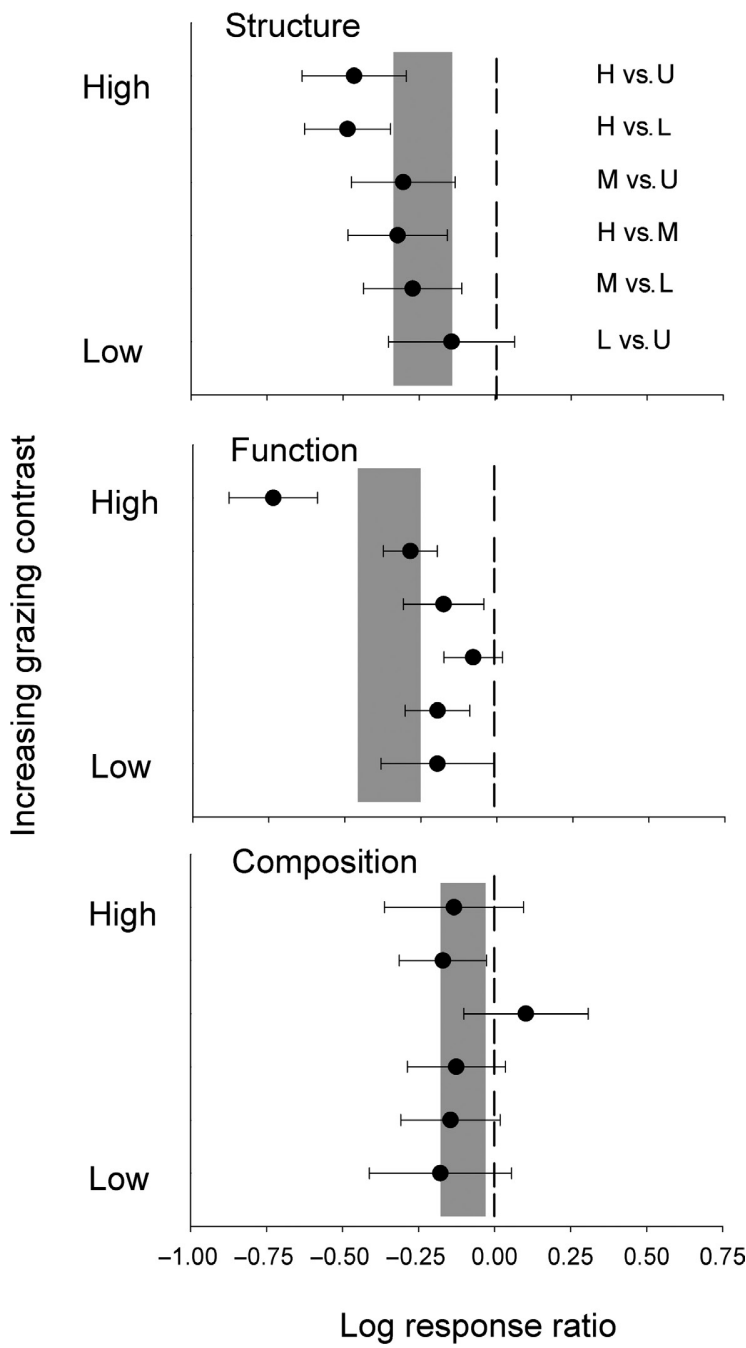

FIG. 2. Estimates $( \pm 95 \% \mathrm{CI})$ of the log response ratio for structure, function, and composition for each of the six grazing contrasts. The shaded region indicates the $95 \%$ confidence interval for the log response ratio with the grazing contrasts pooled. Abbreviations are U, ungrazed; L, lightly grazed; $\mathrm{M}$, moderately grazed; and $\mathrm{H}$, heavily grazed.

structure $\left(\chi^{2}=36.43, \mathrm{df}=2, P<0.01\right)$ and function $\left(\chi^{2}=8.30, \mathrm{df}=2, P<0.05\right)$ declined significantly less in the presence of sheep than when sheep and cattle grazed together (Fig. 3).

Analysis of the responses of broad plant, animal, and soil categories revealed a range of responses to increased grazing, with the effect sizes varying significantly among categories (Fig. 4; $\chi^{2}=32.13$, df $=7, P<0.0001$ ). Plant biomass (function) declined by about $40 \%$, and plant litter and cover (structure) and plant abundance and animal richness (composition) declined by $15-25 \%$ in response to grazing. There were no significant effects, on average, of grazing on plant richness, animal abundance, or soil function (Fig. 4; 95\% confidence intervals overlapping zero).
When these effects were examined in relation to the six grazing contrasts, no category showed a positive response to grazing, though each displayed idiosyncratic responses to increasing grazing pressures (Appendix S4). Plant biomass $\left(\chi^{2}=30.51, \mathrm{df}=5, P<0.0001\right)$ and plant cover $\left(\chi^{2}=15.55, \mathrm{df}=5, P=0.008\right)$ declined linearly in response to increasing grazing contrast. The effect of grazing differed significantly among grazing contrasts for animal richness $\left(\chi^{2}=32.11, \mathrm{df}=5, P<0.001\right)$ and soil function $\left(\chi^{2}=22.23\right.$, df $\left.=5, P<0.001\right)$, but this was mainly due, in both cases, to a suppressive effect at the lowest grazing contrast (Appendix S4).

\section{Effects of aridity on the response of plants, animals, and soils to grazing}

The extent to which differences in rainfall and evaporation (aridity) affected the magnitude of the responses to grazing varied markedly among response variables. Plant biomass declined consistently with increasing grazing in both arid and semi-arid zones (Fig. 5), but in the dry sub-humid zone, grazing significantly reduced biomass only under the highest grazing contrast, with neutral effects under intermediate grazing contrasts (grazing contrast by aridity interaction: $\chi^{2}=24.8$, $\mathrm{df}=10, P=0.007$; Fig. 5).

The interaction between grazing and aridity was less predictable for other response variables. For example, there were some ill-defined effects of grazing on litter cover $\left(\chi^{2}=61.1, \mathrm{df}=4, P<0.001\right)$ and animal abundance $\left(\chi^{2}=5.40, \mathrm{df}=2, P=0.07\right)$ in dry sub-humid zones, and plant abundance $\left(\chi^{2}=10.28, \mathrm{df}=5, P=0.07\right)$ in semi-arid zones (Fig. 5; Appendix S5), but these effects were inconsistent in other zones. Soil function at the highest grazing contrast was significantly lower than that at intermediate grazing contrasts $\left(\chi^{2}=11.39, \mathrm{df}=4, P=0.02\right)$, but only in arid zones (Fig. 5). Similarly, plant cover declined consistently and markedly with increasing grazing contrast in

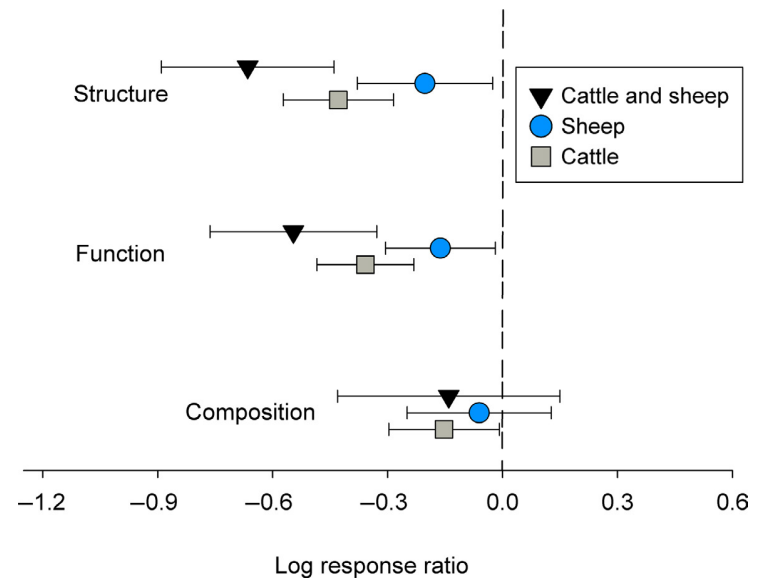

FIG. 3. Estimates $( \pm 95 \% \mathrm{CI})$ of the log response ratio for composition, structure, and function for sheep, cattle, and sheep plus cattle. 


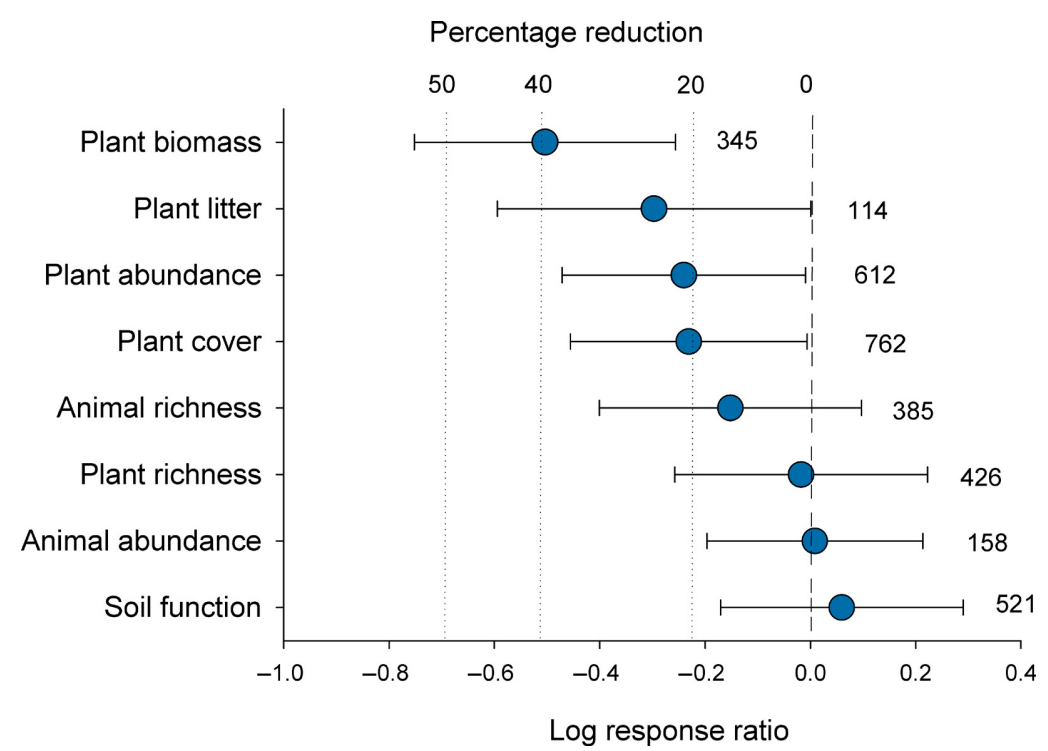

FIG. 4. Estimates ( $\pm 95 \% \mathrm{CI}$ ) of the log response ratio for animal richness and abundance, plant cover, litter, plant abundance and richness, and soil function. The number on the right indicates the number of independent studies.

the arid zone $\left(\chi^{2}=18.30, \mathrm{df}=5, P=0.003\right)$, but effects were largely equivocal in other aridity zones (Fig. 5).

\section{DisCUSSION}

Despite the global distribution of livestock and their importance for sustaining peoples and cultures, there have been relatively few syntheses of grazing-related effects on rangeland ecosystems (Milchunas et al. 1988, Fleischner 1994). Most studies have tended to focus on the response to grazing of a limited suite of variables, often with an emphasis on net primary productivity or community composition (Milchunas et al. 1988, Proulx and Mazumder 1998, van Klink et al. 2014). Our study takes a broader, ecosystem-level approach by examining how livestock grazing affects ecosystem structure, composition and function, using data from Australia. We found mostly negative, but some neutral, responses to grazing, with reductions in our three ecosystem measures under even the most benign levels of grazing. When we examined community attributes associated with plant, animal, and soil categories in more detail, reductions due to grazing ranged from $20 \%$ to $40 \%$. Our study reinforces the notion that grazing effects are largely negative and therefore unequivocal. This is particularly noteworthy considering the continental extent of our analyses, with data compiled from a large number of studies employing different grazing systems and herbivores, and spanning multiple geographical domains, years, and seasonal conditions. The implications of our work are that, overall, grazing by livestock in Australia is unlikely to result in positive outcomes for ecosystem structure, composition, and function.
Compositional, structural, and functional responses to grazing

In our study, grazing had a slightly negative effect on composition (e.g., richness, abundance, diversity), which remained relatively constant with increasing grazing pressure, irrespective of herbivore type. Greater levels of livestock grazing can generate substantial shifts in plant and animal composition. However, the relatively modest effects we found can be explained by the weak influence of grazing on the richness component of the compositional metric, probably due to idiosyncratic responses of many different taxa to grazing. For example, intensive grazing associated with agricultural activities has been shown to substantially reduce richness of plants, lichens, and insects (e.g., Orthoptera and Lepidoptera), but not Diptera, arbuscular mycorrhizal fungi or bats (Allan et al. 2014). Grazing-induced increases in ants of the dominant Dolichorine group could buffer reductions in cryptic, grazing-sensitive species, resulting in no net change in richness (Bromham et al. 1999, Seymour and Dean 1999, Nash et al. 2004). Moderate grazing may increase plant richness by removing highly competitive species and increasing light availability (Borer et al. 2014). However, while this may be true of productive grasslands (Socher et al. 2013, Borer et al. 2014), our extensive meta-analysis casts doubt on this as a general response. Most of our studies come from arid and semiarid environments in Australia, so that, for plant species richness, there was little benefit of moderate grazing (Fig. 5; Appendix S5).

The mechanism underlying compositional changes is almost certainly an indirect effect related to structural changes to vegetation, such as cover, complexity, and plant height (van Klink et al. 2014). In our study, even 
Plant biomass

Arid
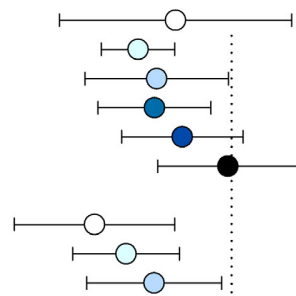

Semi-arid

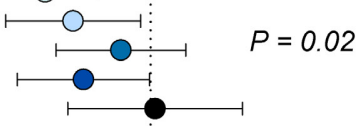

Dry sub-humid
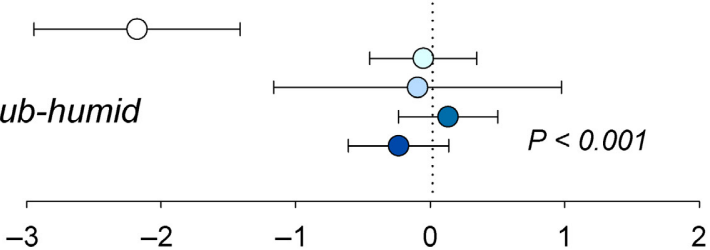

Litter cover

Arid

Dry sub-humid
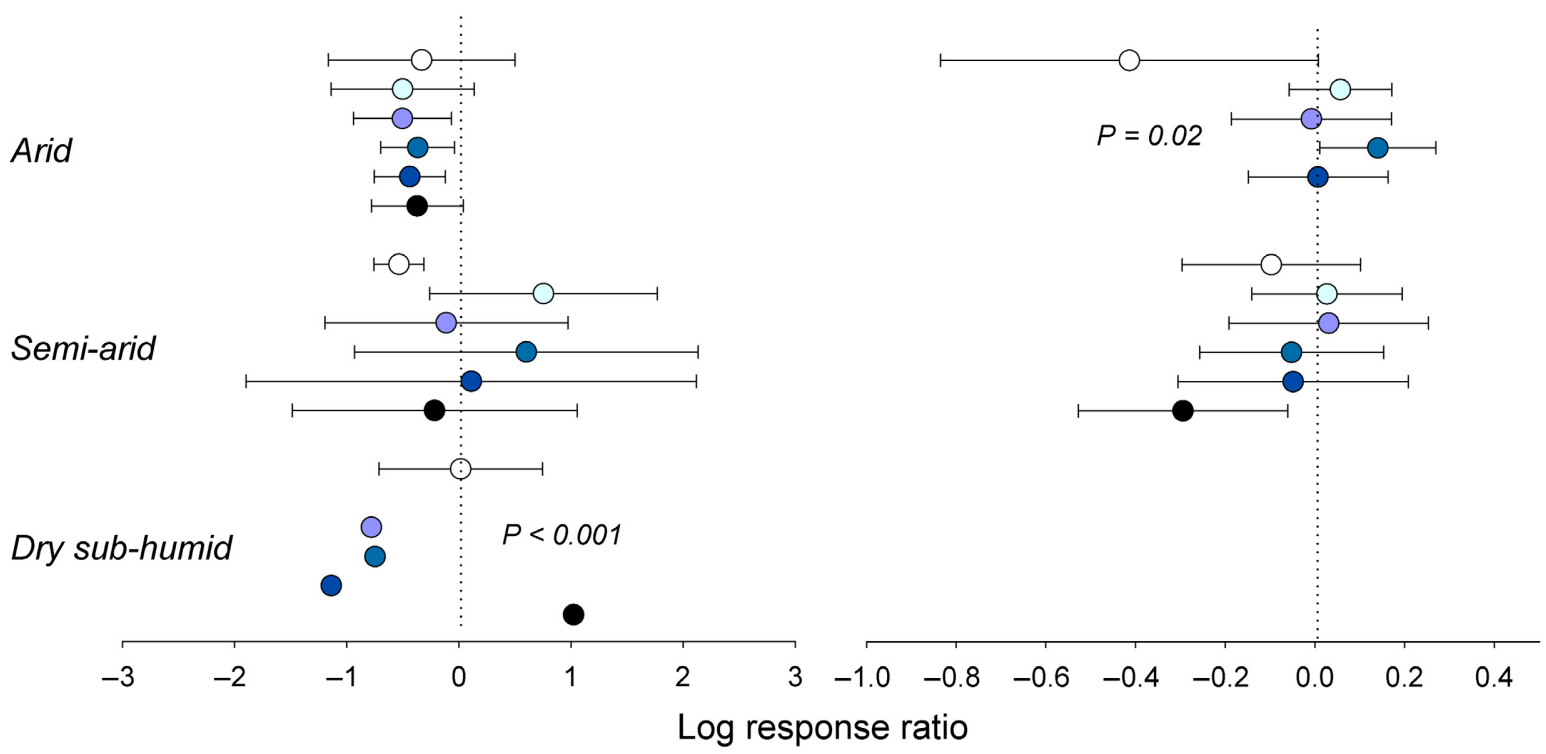

\section{Plant cover}

H vs.U

H vs.L

$M$ vs. U

H vs. M

$M$ vs. L

L vs.U

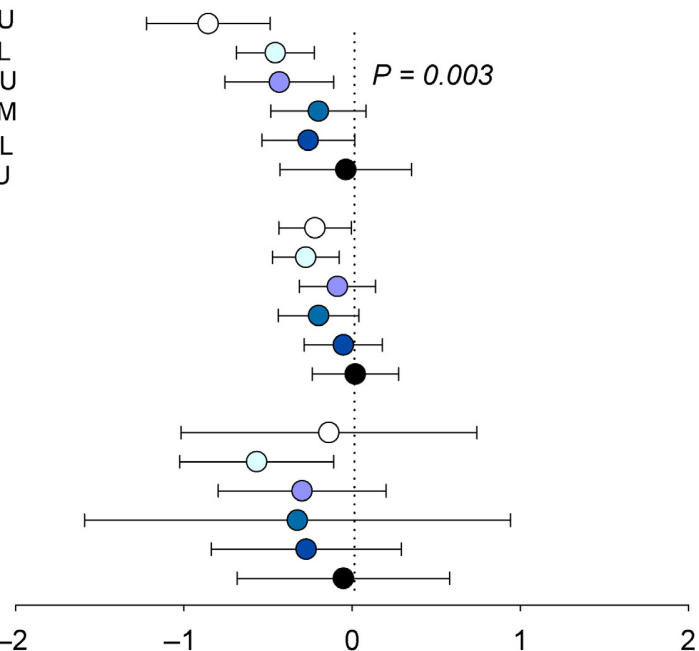

FIG. 5. Estimates $( \pm 95 \% \mathrm{CI})$ of the log response ratios for plant biomass, plant cover, litter cover, and soil function by grazing contrast and aridity class. Note: there are no data for soil function for dry sub-humid areas. Abbreviations are U, ungrazed; L, lightly grazed; M, moderately grazed; and $\mathrm{H}$, heavily grazed. $P$ values are presented where the six grazer contrasts were significantly different.

very low levels of grazing reduced structure, which declined consistently with increasing grazing contrast (Fig. 2). Grazing typically selects for shorter, prostrate annual plants at the expense of taller perennials and annual grasses (Noy-Meir et al. 1989), or plants with spines, waxy leaves, or higher levels of secondary compounds (Milton et al. 1994). Under very low grazing pressure, open habitat specialists are more likely to disappear. For example, the Plains Wanderer (Pedionomus torquatus), a threatened ground-dwelling bird from eastern Australia, benefits from substantial areas of bare ground generated by sheep grazing (Parker and Oliver 2006). The gain of these open habitat species can cancel out the loss of the most grazing sensitive species resulting in no net effect of grazing on animal diversity under intermediate grazing pressures (Appendix S5). 
There were two main effects of grazing on function in our study. First, functional effects were mediated mainly by changes in plant biomass, but not by soils. Biomass was reduced by about $40 \%$, but for soil function, there were a mixture of positive and negative effects across grazing contrasts (Fig. 4). The index of soil function comprised measures of soil carbon, nitrogen, and phosphorus; slow variables linked to key soil functional processes that are likely to be affected by grazing (Milchunas and Lauenroth 1993). The negative effects on some functional variables could be matched, however, by increases in organic matter and the products of nitrogen deposition, through excretion of dung and urine, resulting in average functional responses close to zero (Fig. 5). While these overall neutral effects are likely across a large range of grazing contrasts, we found that this changes at the highest (heavy vs. ungrazed) contrast where both soil function and biomass declined markedly (Fig. 5). Threshold levels in function, such as those observed for biomass, have been demonstrated for soil chemistry and plant biomass under very heavy levels of grazing in piosphere around livestock watering points in Australia (Andrew and Lange 1986). Marked declines in function would have substantial flow-on effects to landscape-level nutrient redistribution. Under intermediate grazing contrasts in commercial grazing systems, large amounts of nitrogen would be returned to the soil. At heavy levels of grazing and surface disturbance, however, this would reduce soil function as the $\mathrm{P}: \mathrm{N}$ ratio increases and phosphorus becomes decoupled from nitrogen (DelgadoBaquerizo et al. 2013).

\section{Grazing effects vary with herbivore type}

The effects of cattle on structure and function ( $40 \%$ reduction) were more negative than those due to sheep ( $\sim 14 \%$ reduction), though the overlap in confidence intervals indicates a high degree of variability among herbivores and studies. The more negative impact from cattle grazing could be due to differences in foraging patterns, dentition, and plant preference. Sheep are able to crop vegetation closer to the ground than cattle, which have no upper incisors; hence groundstory vegetation tends to be shorter in sheep than cattle pastures (Squires 1980). Further, the tendency of cattle to eat taller grasses, often more fibrous and less digestible plants (Squires 1981) and to uproot shallow-rooted plants (Letnic 2004) may also account for their greater influence on vegetation structure than sheep. Overall, sheep and cattle vary in their preferences for different species, graze differently, and therefore have a synergistic effect on vegetation structure.

\section{Grazing effects are moderated by rainfall}

The interactions between grazing effects and productivity have been hotly debated for decades (e.g., Milchunas and Lauenroth 1993, Proulx and Mazumder 1998, Cingolani et al. 2005). Our study showed that these contrasting views stem from the different response variables and grazing contrasts on which these studies focus. Negative effects of grazing were generally more pronounced for plant cover, biomass and soil function in less productive (arid) systems (Fig. 5). Interestingly, this general trend changed under the highest grazing contrast, where the decline in biomass was greatest in the wettest areas. Our results also suggest that litter cover may follow a similar trend to plant cover. The low number of studies, however, particularly from dry sub-humid areas, and the large confidence intervals prevented us from detecting significant trends in most response variables.

In contrast to the traditional view (e.g., Proulx and Mazumder 1998), grazing effects remained unchanged (plant and animal abundance) or declined slightly (plant and animal richness) with increasing aridity. Some of this may relate to differences in herbivore composition, with sheep grazing (which predominates in dry environments) known to have some positive effects on plant richness (Socher et al. 2013). As indicated previously, most of the effects of grazing on composition were mediated through structural simplification of the habitat. As structural effects are likely to be less evident in drier areas because of the sparser vegetation, it is reasonable to assume that effects on plant and animal abundance and richness should also be less pronounced in such environments.

\section{Methodological consideration: limitations to our study}

In addition to livestock, the Australian rangelands are also grazed by considerable populations of wild herbivores including kangaroos (Macropus spp.), rabbits (Oryctolagus cuniculus), feral goats (Capra hircus), feral horses (Equus caballus), feral donkeys (Equus asinus), camels (Camelus dromedarius), and more recently, deer (Cervus spp., Axis spp.), but little is known of their impacts on ecosystems. Of these, kangaroos and rabbits are most abundant and widespread. Very few of the studies identified in our literature search (e.g., Bridle and Kirkpatrick 1999) included kangaroo or rabbit grazing as a single treatment, and there were insufficient data on which to base an assessment of their ecosystem effects relative to livestock effects. Further, almost all of our studies of the effects of sheep grazing occurred in areas where dingo (Canis dingo) populations are suppressed through fencing, poisoning, or shooting (Letnic and Crowther 2013). Kangaroo populations typically irrupt in areas where dingoes are suppressed resulting in dramatic increases in total grazing pressure and the depletion of their preferred forage, grasses (Norbury et al. 1993, Letnic et al. 2009). The paucity of sheep grazing studies in the presence of dingoes precluded us from using the presence of dingoes as a control for this residual grazing, which would have dampened the grazing effect we encountered. Furthermore, a relatively large number of sites included livestock-proof exclosures, which we used to derive an assessment of the ungrazed control. It is possible, however, that in some situations, kangaroos were able to gain access to livestock-proof exclosures if fences 
were not sufficiently high and/or robust to exclude kangaroos (Eldridge et al. 1990). Indeed, studies have shown that kangaroos tend to congregate in areas where livestock have been excluded, presumably to utilize ungrazed pastures (Norbury et al. 1993). Although this limitation of our study should be considered when interpreting the results, residual grazing is unlikely to significantly influence our results. Our main results were consistent both for the three grazed-ungrazed comparisons (i.e., ungrazed cf. light, medium, or heavy), and for the three intermediate levels of grazing, all accessible to potential kangaroo grazing (i.e., light cf. moderate, light cf. heavy, moderate cf. heavy; Appendix S3). This suggests that moderate levels of kangaroo grazing in the control treatments (e.g., ungrazed vs. light grazing) are unlikely to affect our main conclusions. Interpretation of livestock effects from these experiments would also be complicated if ungrazed is no livestock or kangaroos and grazed is livestock and kangaroos. The differences found between cattle and sheep suggest that livestock are the main determinants of grazing effects, as both would also have residual grazing by kangaroos.

\section{Concluding remarks}

Overall, our study showed that livestock grazing consistently reduced indices of ecosystem structure, function, and composition across a large area of Australia. Further, herbivore type did not influence composition, but both structure and function were reduced significantly when both sheep and cattle graze together compared with either grazing alone. Predictably, the greatest effect of grazing was on biomass, with average declines of about $40 \%$, but both increases in declines in plant richness, animal abundance, and soil function with grazing were equally probable. Our most striking result was that even the lowest grazer densities negatively affected ecosystems, suggesting that, for attributes such as plant litter, plant abundance, plant cover, and animal richness, even low levels of grazing will result in significant declines in these variables. Low levels of grazing are therefore unlikely to be a useful tool for managing ecosystems unless reductions in these response variables are an explicit management objective. We see strong potential for similar meta-analyses focused on other regions. Australia has a short evolutionary history of grazing by domestic livestock, so grazing is less likely to have large positive effects on ecosystem processes compared with other environments such as Southern Africa. The negative effects of grazing were sometimes most pronounced in arid environments as commonly accepted, although this grazing by productivity interaction was much less likely for compositional variables.

\section{ACKNOWLEDGMENTS}

We thank many colleagues who provided access to data and information from vegetation exclosures. M. Ruiz-Colmenero was supported by a grant from EU's Cooperation on Science and Technology (COST) Program (COST ES1104 Restoration and Desertification Rehabilitation Hub). We appreciate useful discussions with Ian Lunt, Manuel Delgado-Baquerizo, Ian Oliver, and Josh Dorrough.

\section{Literature Cited}

Allan, E., et al. 2014. Interannual variation in land-use intensity enhances grassland multidiversity. Proceedings of the National Academy of Sciences USA 111:308-313.

Andrew, M. H., and R. T. Lange. 1986. Development of a new piosphere in arid chenopod shrubland grazed by sheep. 2: changes to the vegetation. Australian Journal of Ecology 11:411-424.

Bates, D., M. Maechler, B. Bolker, and S. Walker. 2014. lme4: linear mixed-effects models using Eigen and S4. R package version 1.1-7. http://CRAN.R-project.org/package=lme4

Borer, E. T., et al. 2014. Herbivores and nutrients control grassland plant diversity via light limitation. Nature 508:517-520.

Bridle, K. L., and J. B. Kirkpatrick. 1999. Comparative effects of stock and wild vertebrate herbivore grazing on treeless subalpine vegetation, Eastern Central Plateau, Tasmania. Australian Journal of Botany 47:817-834.

Bromham, L., M. Cardillo, A. F. Bennett, and M. A. Elgar. 1999. Effects of stock grazing on the ground invertebrate fauna of woodland remnants. Australian Journal of Ecology 24:199-207.

Cingolani, A. M., I. Noy-Meir, and S. Díaz. 2005. Grazing effects on rangeland diversity: a synthesis of contemporary models. Ecological Applications 15:757-773.

Delgado-Baquerizo, M., et al. 2013. Decoupling of soil nutrient cycles as a function of aridity in global drylands. Nature 502:672-676.

Dorrough, J., S. McIntyre, G. Brown, J. Stol, G. Barrett, and A. Brown. 2012. Differential responses of plants, reptiles and birds to grazing management, fertilizer and tree clearing. Austral Ecology 37:569-582.

Eldridge, D. J., M. Westoby, and R. J. Stanley. 1990. Population dynamics of the perennial rangeland shrubs Atriplex vesicaria, Maireana astrotricha and M. pyramidata under grazing. Journal of Applied Ecology 27:502-512.

Eldridge, D. J., M. A. Bowker, F. T. Maestre, E. Roger, J. F. Reynolds, and W. G. Whitford. 2011. Impacts of shrub encroachment on ecosystem structure and functioning: towards a global synthesis. Ecology Letters 14:709-722.

Eldridge, D. J., S. Soliveres, M. A. Bowker, and J. Val. 2013. Grazing dampens the positive effects of shrub encroachment on ecosystem functions in a semi-arid woodland. Journal of Applied Ecology 50:1028-1038.

Fensham, R. J., J. L. Silcock, and J. Firn. 2014. Managed livestock grazing is compatible with the maintenance of plant diversity in semidesert grasslands. Ecological Applications 24:503-517.

Fleischner, T. L. 1994. Ecological costs of livestock grazing in western North America. Conservation Biology 8:629-644.

Gurevitch, J., and L. V. Hedges. 1999. Statistical issues in ecological meta-analyses. Ecology 80:1142-1149.

Kimuyu, D. M., R. L. Sensenig, C. Riginos, K. E. Veblen, and T. P. Young. 2014. Native and domestic browsers and grazers reduce fuels, fire temperatures, and acacia ant mortality in an African savanna. Ecological Applications 24:741-749.

van Klink, R., F. van der Plas, C. G. E. van Noordwijk, M. F. WallisDeVries, and H. Olff. 2014. Effects of large herbivores on grassland arthropod diversity. Biological Reviews 90:347-366.

Landsberg, J., C. D. James, S. R. Morton, W. J. Muller and J. Stol. 2003. Abundance and composition of plant 
species along grazing gradients in Australian rangelands. Journal of Applied Ecology 40:1008-1024.

Letnic, M. 2004. Cattle grazing in a hummock grassland regenerating after fire: the short-term effects of cattle exclusion on vegetation in south-western Queensland. Rangeland Journal 26:34-48.

Letnic, M., and M. S. Crowther. 2013. Patterns in the abundance of kangaroo populations in arid Australia are consistent with the exploitation ecosystems hypothesis. Oikos 122:761-769.

Letnic, M., F. Koch, C. Gordon, M. S. Crowther, and C. R. Dickman. 2009. Keystone effects of an alien top-predator stem extinctions of native mammals. Proceedings of the Royal Society B 276:3249-3256.

Lezama, F., S. Baeza, A. Altesor, A. Cesa, E. J. Chaneton, and J. M. Paruelo. 2014. Variation of grazing-induced vegetation changes across a large-scale productivity gradient. Journal of Vegetation Science 25:8-21.

Lunt, I. D., D. J. Eldridge, J. W. Morgan, and G. B. Witt. 2007. A framework to predict the effects of livestock grazing and grazing exclusion on conservation values in natural ecosystems in Australia. Australian Journal of Botany 55:401-415.

Lunt, I. D., A. Jansen, and D. Binns. 2012. Effects of flooding and livestock grazing on exotic annual plants in riverine floodplains. Journal of Applied Ecology 49: 1131-1139.

Milchunas, D. G., and W. K. Lauenroth. 1993. Quantitative effects of grazing on vegetation and soils over a global range of environments. Ecological Monographs 63:327-366.

Milchunas, D. G., O. E. Sala, and W. Lauenroth. 1988. A generalized model of the effects of grazing by large herbivores on grassland community structure. American Naturalist 132:87-106.

Milton, S. J., W. R. J. Dean, M. A. du Plessis, and W. R. Siegfried. 1994. A conceptual model of arid rangeland degradation. BioScience 44:70-76.

Møller, A. P., and M. D. Jennions. 2001. Testing and adjusting for publication bias. Trends in Ecology and Evolution 16:580-586

Mooney, K. A., D. S. Gruner, N. A. Barber, S. A. Van Baeld, S. M. Philpott, and R. Greenberg. 2010. Interactions among predators and the cascading effects of vertebrate insectivores on arthropod communities and plants. Proceedings of the National Academy of Sciences USA 107:7335-7340.

Nash, M. S., D. F. Bradford, S. E. Franson, A. C. Neale, W. G. Whitford, and D. T. Heggem. 2004. Livestock grazing effects on ant communities in the eastern Mojave Desert, USA. Ecological Indicators 4:199-213.

Norbury, G. L., D. C. Norbury, and R. B. Hacker. 1993. Impact of red kangaroos on the pasture layer in the Western Australian arid zone. Rangeland Journal 15:12-23.

Noss, R. F. 1990. Indicators for monitoring biodiversity: a hierarchical approach. Conservation Biology 4:355-364.
Noy-Meir, I., M. Gutman, and Y. Kaplan. 1989. Responses of Mediterranean grassland plants to grazing and protection. Journal of Ecology 77:290-310.

Parker, D. G., and D. L. Oliver. 2006. Plains-wanderer habitat management guide. A photographic guide for visually assessing the grassland structure of plains-wanderer habitat. Second edition. Department of Environment and Conservation, Sydney, New South Wales, Australia.

Piñeiro, J., F. T. Maestre, L. Bartolomé, and A. Valdecantos. 2013. Ecotechnology as a tool for restoring degraded drylands: a meta-analysis of field experiments. Ecological Engineering 61:133-144.

Poore, A. G., et al. 2012. Global patterns in the impact of marine herbivores on benthic primary producers. Ecology Letters 15:912-922.

Proulx, M., and A. Mazumder. 1998. Reversal of grazing impact on plant species richness in nutrient-poor vs. nutrientrich ecosystems. Ecology 79:2581-2592.

Riginos, C., and J. B. Grace. 2008. Savanna tree density, herbivores, and the herbaceous community: bottom-up vs. top-down effects. Ecology 89:2228-2238.

Senft, R. L., M. B. Coughenour, D. W. Bailey, L. R. Rittenhouse, O. E. Sala, and D. M. Swif. 1987. Large herbivore foraging and ecological hierarchies. BioScience 36:789-799.

Seymour, C. L., and W. R. J. Dean. 1999. Effects of heavy grazing on invertebrate assemblages in the Succulent Karoo, South Africa. Journal of Arid Environments 43:267-286.

Socher, S. A., D. Prati, S. Boch, J. Müller, H. Baumbach, S. Gockel, and M. Fischer. 2013. Interacting effects of fertilization, mowing and grazing on plant species diversity of 1500 grasslands in Germany differ between regions. Basic and Applied Ecology 14:126-136.

Squires, V. R. 1980. Chemical and botanical composition of the diets of oesophageally fistulated sheep, cattle and goats in a semi-arid Eucalyptus populnea woodland community. Australian Rangeland Journal 2:94-103.

Squires, V. R. 1981. Livestock management in the arid zone. First edition. Inkata Press, Melbourne, Victoria, Australia. Tongway, D. J. 1995. Monitoring soil productive potential. Environmental Monitoring and Assessment 37:303-318.

Tummers, B. 2006. DataThief III. http://datathief.org

United Nations Environment Programme. 1992. World atlas of desertification/UNEP, United Nations Environment Programme. Second edition. Edward Arnold, London, UK. Williams, R. J., C. H. Wahren, R. A. Bradstock, and W. J. Muller. 2006. Does alpine grazing reduce blazing? A landscape test of a widely-held hypothesis. Austral Ecology 31:925-936.

Williams, W. J., D. J. Eldridge, and B. M. Alchin. 2008. Grazing and drought reduce cyanobacterial soil crusts in an Australian Acacia woodland. Journal of Arid Environments 72:1064-1075.

Williamson, G. J., B. P. Murphy, and D. M. J. S. Bowman. 2014. Cattle grazing does not reduce fire severity in eucalypt forests and woodlands of the Australian Alps. Austral Ecology 39:462-468.

\section{SUPPORTING INFORMATION}

Additional supporting information may be found in the online version of this article at http://onlinelibrary.wiley.com/ doi/10.1890/15-1234.1/suppinfo 